\title{
Medical image analysis and 3-d modeling to quantify changes and functional restoration in denervated muscle undergoing electrical stimulation treatment
}

Paolo Gargiulo ${ }^{1,2^{*}}$, Thordur Helgason ${ }^{1,2}$, Páll Ingvarsson ${ }^{3}$, Winfried Mayr ${ }^{4}$, Helmut Kern $^{5}$ and Ugo Carraro ${ }^{6}$

\author{
*Correspondence: paologar@ \\ landspitali.is \\ 'Department of Development and \\ Consultancy HUT, University \\ Hospital Landspitali, Armuli 1A \\ Reykjavik 108, Iceland \\ ${ }^{2}$ Department of Biomedical \\ Engineering, University of Reykjavik, \\ Armuli 1A, Reykjavik 108, Iceland \\ Full list of author information is \\ available at the end of the article
}

\begin{abstract}
Background and purpose: Muscle tissue composition can be efficiently analyzed and quantified using images from spiral computed tomography technology (SCT) and the associated values of Hounsfield unit (HU) for different tissues. This work propose a novel approaches to monitor muscle condition in denervated muscle undergoing electrical stimulation (ES) treatment based on image segmentation and Three Dimensional (3D) modeling.

Method: Three paraplegic patients with fully denervated muscles in the lower extremities were treated with ES. To follow changes in size, composition and shape of the quadriceps muscle, SCT scans are taken every 6 months from the trochanter major to the knee for 4 years. Using segmentation techniques we isolated rectus femoris muscle (RF) and analyzed its content of fat, connective, and muscle tissue.

Results: The results showed the muscle restoration and growth induced by ES. The amount of normal muscle fibers increased from $45 \%$ to $60 \%$ of the whole volume while connective tissue and fat was reduced respectively of $30 \%$ and $50 \%$. It was also found that muscles undergoing ES were restored in certain areas while declined in others depending on patient's anatomy and positioning the surface electrodes.

Conclusion: The 3D approach combined with muscle tissue analysis provides information on the whole muscle and on its structural changes during ES treatment otherwise not accessible with other monitoring techniques.
\end{abstract}

Keywords: Electrical Stimulation, Image Segmentation, Denervated Muscle, Muscle Fiber, Spinal Cord Injury

\section{Springer}

\section{Introduction}

Loss of muscle mass occurs with many pathological conditions and it is linked to increased patient disability, morbidity and mortality. Thus, it is important to find ways to deter muscle degeneration [1,2]. Empirical clinical observations have show that the lower motor neuron (LMN) denervated degenerated muscle can recover by a specific variation of home based daily functional electrical stimulation (ES) therapy [3]. This is in contradiction to an earlier data which suggested that ES was effective only when started immediately after LMN lesion. These observations have led to the funding of

(c) 2012 Gargiulo et al.; licensee Springer. This is an Open Access article distributed under the terms of the Creative Commons Attribution License (http://creativecommons.org/licenses/by/2.0), which permits unrestricted use, distribution, and reproduction in any medium, provided the original work is properly cited. 
the European funded project RISE in November 2001. The project's aim was to establish the biological basis for a clinical rehabilitation treatment for patients who have permanent muscle LMN denervation in the lower extremities. To this end, it funded research designed to reverse muscle degeneration induced by the permanent lack of innervation in spinal cord injured (SCI) patients using muscle ES. Some of the funds have been used to carry out research in rehabilitative centres in Vienna (Austria), Heidelberg (Germany), Hamburg (Germany), Tübingen (Germany), Reykjavik (Iceland) and Vicenza (Italy). The RISE project has achieved its goal using a multidisciplinary approach to optimize technology to stimulate LMN denervated muscle with custom-designed electrodes and stimulators developed in Vienna, Austria [4,5].

The project encompassed a clinical trial with over 25 voluntary patients and additional animal experiments to research the muscle restoration process by combining physiological, histological, immunohistochemical, and biochemical analyses with anthropometric techniques $[6,7]$.

The results of the EU RISE Project, and of the related animal research, provide different perspectives.

Twenty out of 25 patients completed a 2 years h-b (home based) ES program [4,8], which resulted in:

1. Significant increase of thigh muscle size and of the muscle fibers, with striking improvements of the ultra-structural organization of contractile material.

2. Significant increase in muscle force output during ES (knee extension torque).

3. The recovery of quadriceps muscle force was sufficient to allow compliant subjects to perform ES-assisted stand-up and stepping-in-place exercises.

4. Ultra structural analyses demonstrated that the shorter was the time elapsed from $\mathrm{SCI}$ to the beginning of h-b ES, and the size of recovered fibers were larger in numbers.

The study demonstrates that h-b ES of permanent LMN denervated muscle is an effective home therapy that results in rescue of muscle mass and tetanic contractility. Important benefits for the patients are the improved cosmetic appearance of lower extremities, the enhanced cushioning effect for seating and the early result of impressive reduction of the leg edema $[9,10]$. The last observation is supported by changes of the capillary networks observed in the muscle biopsies harvested from subjects suffering with long-lasting LMN denervation before and after h-b ES and thoracic level SCI [11].

Many of the tissue analyses employed to study structural changes occurring in LMN denervated muscle (both after long term LMN denervation and during ES) were performed with biopsies which meant that only a few milligrams of muscle could be analyzed [8]. Stimulation needle electromyography was used to study the muscle fibre conduction velocity and refractory period. The patients performing electrical stimulation training showed higher conduction velocities and reduced refractory periods despite of longer lasting denervation [12] . Complementary imaging techniques, such as X-ray computed tomography (CT), were also employed in order to assess and validate histological information. Muscle complete cross-sectional area and density (measured in $\mathrm{HU}$ ) of quadriceps muscle and hamstrings were determined as described in Mödlin et al [7]. The value of the imaging methods demonstrates that the development and use of non-invasive anthropometric techniques is critical to this area of research. 


\section{Material and methods \\ Patients}

Pre-selection of patients was performed at the Department of Rehabilitation Medicine, Landspitali-University Hospital, Reykjavik, Iceland. In the present study data from two males suffering from Conus Cauda Syndrome at 1 year (subject 1, 30 years old) and 4 years post SCI (subject 2, 25 years old) was used. These subjects participated in home based ES according to Kern [3]. Computed Tomography scans of the lower extremities are carried out at the Department of Research and Development, HTS, Landspitali-University Hospital, Reykjavik, Iceland. Clinical and functional assessments for the enrollment of the patients in the EU project RISE, as well as follow-up and muscle biopsies, were performed at the Wilhelminenspital, Vienna, Austria. Light microscopy analyzes were performed at the University of Padova, Italy. For enrollment inclusion and exclusion criteria see Table 1 of [8].

Patients were supposed to stimulate their lower extremities 5-6 times a week according to the RISE stimulation protocol but this schedule wasn't respected all the time: Subject 1 was compliant in the first year but stimulating poorly and not regularly for the rest of the time while Subject 2 was stimulating regularly and intensively over the observed period. Muscle and bone progression were monitored taking spiral CT scanners 3-4 times per year.

As a consequence of long term denervation the muscle degenerates dramatically. The muscles become very thin and the single bellies are no longer recognizable in their shape. Only rectus femoris (RF) remains recognizable among the quadriceps muscles though it is severely degenerated compared to the normal situation. The large surface electrodes employed during electrical stimulation treatment are placed on top of the quadriceps muscles, RF has a central position within the muscle group and therefore especially exposed to the current distribution [14].

$\mathrm{RF}$ is thus the optimal target for monitoring therapeutical effects and morphological changes with a 3D approach $[15,16]$.

3D modeling and segmentation techniques used here aim to isolate RF from other muscle bellies and to monitor changes occurring during the degeneration and restoration process.

\section{Data set source}

$\mathrm{X}$-ray computed tomography is an imaging method that uses X-rays to produce images of structures 'inside' the body. Patients are scanned slice-per-slice and each slice is scanned several times from different angles. Each imaged volume element (voxel) is traversed, during

Table 1 The table displays the main human tissues and their empirical HU intervals [13]

\begin{tabular}{lll}
\hline Anatomical Tissues & Hounsfield intervals \\
\cline { 2 - 3 } & Min & 3071 \\
\hline Bone & 250 & 1988 \\
Compact bone & 601 & 600 \\
Spongy bone & 250 & 80 \\
Normal Muscle & 41 & 200 \\
Dense fibrous connective (Tendons-dense muscle) & 81 & 40 \\
Loose connective (low dense muscle) & -5 & -6 \\
Fat & -200 & 60 \\
Skin & -30 & 3071 \\
Tooth & 1200 & \\
\hline
\end{tabular}


the scan, by numerous X-ray photons and the intensity of the transmitted radiation is measured by detectors. The measured intensity profile contains information on the densities the beam encountered on its path through the body (i.e. the denser the regions the weaker the signal). With suitable mathematical methods the measured profiles of each slice are transformed into an image of the structures inside - the image is reconstructed. This way, 3Dimensional data are gathered scanning the patient's lower limbs with spiral CT. The scan starts above the head of the femur and continues down to the knee joint. Both legs were scanned in one session. Slice increment is set to $0.625 \mathrm{~mm}$ resulting in a total of about 750-900 CT slices, depending on the patient's size.

Every acquired CT slice is subdivided into a matrix of different size, from a minimum of $128 \times 128$ up to $1024 \times 1024$ volume elements. The average linear attenuation coefficient, $\mu$ of the tissue contained in each voxel is represented by floating numbers in the computer which range from 0.0 up to values equal to 1.0.

Once the image is calculated from the 3D data set it is converted into a matrix of picture elements (pixels) with each pixel assigned the attenuation value of the corresponding voxel. Linear attenuation coefficients are rescaled to an integer range that encompasses 4096 values, spread between -1000 and 3095 . From these intensity readings, the density or attenuation value of the tissue at each point in the slice can be calculated. This scale is called CT number or Hounsfield unit (HU) and it is expressed by the following formula (1):

$$
\text { CTNumber }=1000 \times \frac{\mu \text { pixel }-\mu \text { water }}{\mu \text { water }}
$$

With this scaling, if the linear attenuation coefficient of a given pixel is equal to that of water, the CT number will be 0 . If is less than $\mu$ water the CT number will be negative which is typical for air spaces, lung tissues and fatty tissues. Values of greater than will result in positive CT numbers. Very dense tissue such as bone has large positive numbers. Table 1 displays main organic tissues and respective HU intervals calculated experimentally [13].

\section{Image processing}

Segmentation process begins by establishing a threshold, which discriminates the region of interest from the rest by selecting appropriate ranges of gray values (GV). From the visual point of view thresholding allows highlighting (for example in different colors) pixels with certain gray values from the others. In an $\mathrm{M} \times \mathrm{N}$ slice, each element in the image matrix $a$ $[m, n]$ displays a level of brightness coded by a grey value which in medical imaging varies from 0 to $4095\left(=2^{12}-1\right)$. For example, the region of interest to be visualized is between GVmin to GVmax then the threshold test condition will appear as in (1):

$$
\begin{aligned}
& \text { If GVmin }<=a[m, n]<=\text { GVmax then } a[m, n]=\text { object }=\text { color } \\
& \text { Else } a[m, n]=\text { background }
\end{aligned}
$$

After thresholding further segmentation tools are usually required to isolate ROI from surrounding areas. Region growing for example is a segmentation tools used to eliminate floating pixels from belonging to the selected threshold. It is often used when determining pixel class membership: pixels that belong to the same region are connected. Other operations such as Boolean and morphological are possible on defined pixel classes in order to improve the segmentation work. Particularly convenient in the segmentation of CT based 
image is the use of specific HU values associated to the pixels rather than the GV because it can be directly related to type of biological tissue.

\section{Segmentation of RF}

The threshold interval chosen to segment RF is: $[-5,200]$ HU. Muscle fibers are mostly displayed with $\mathrm{HU}$ values between 40 and 80, a wide interval is chosen here because it must include all the tissues within the muscle volume. In this way will be possible to monitor changes occurring during stimulation treatment, particularly the restoration-degeneration process. Within the selected interval the displayed pixels represents normal and degenerated muscles, connective tissues and water, but excludes subcutaneous fat. In this way the surrounding fat in RF is automatically excluded from the segmentation mask but not the other muscles such as vastus lateralis, vastus medialis and vastus intermedius. There isn't automatic segmentation methods that allow the discrimination of rectus femoris from the other quadriceps muscles, only the anatomy of the thigh and use of semiautomatic segmentation tools can help for this purpose. After thresholding, the next step for the segmentation is to isolate RF form the other muscles. For this purpose the following procedure is used. The process starts from a cross section where the muscle boundaries are well defined and visible usually in the middle, along the length of the muscle. A contour is manually drawn around the muscle and projected to the next cross sections in both directions. If the contour fits the new cross sectional area well then it is projected unchanged forward to the next slice, otherwise it is adapted and then projected ahead. We assume that shapes change little from one slice to the next and the necessary adaptation is very small. It is done with active contours that 'snap' to boundaries.

The process continues until all cross sections containing RF cross sections are edited. The contour areas are then erased creating a gap between segmentation target and surrounding. Finally, a new segmentation mask representing RF is created by applying a region growing procedure which creates a new mask separating the edited structure that is no longer connected to the surrounding.

All Segmentation was performed with software MIMICS 14.1 [17].

\section{Muscle tissue analysis}

In order to discriminate the biological tissues in the data set, different thresholds are established using the HU scale. Specific attenuation values are assigned to each individual voxel. The degree of attenuation depends on the energy spectrum of the $\mathrm{x}$-rays as well as on the average atomic number of the mass density of the patient tissue. Most computer display hardware requires integer numbers and therefore the linear attenuation coefficients are rescaled to an integer range that encompasses 4096 values, spread between -1000 and 3095. Dense tissue such as bone has large positive CT number while negative CT numbers are typical for air spaces, lung tissues and fatty tissues. Muscle tissues are normally displayed with HU values between 50 and $100 \mathrm{HU}$ though within a normal muscle belly there are also other tissue elements such as connective and fat which are coded with much lower HU values. Anyway the specific HU value depends also on the pixel size. Indeed every element can express its absolute $\mathrm{HU}$ value if it occupies completely the specific pixel volume otherwise this value will be an average between the different parts contained in it. This fact explains the wide range of 
values present inside a data set and suggests the definition of various intervals to study muscle structural changes. Therefore to monitor and quantify tissue composition in the stimulated muscle volume the $\mathrm{HU}$ values present within the segmented volume are divided in four HU intervals $[-200,-6],[-5,-40],[41,80]$ and $[81,200]$ representing respectively fat, loose connective (low dense muscle), normal muscle and Dense fibrous connective (Tendons-dense muscle).

\section{Cross sectional density analysis}

In order to evaluate the effectiveness of the ES treatment using surface electrodes we develop a Matlab (Matworks Inc) subroutine to analyze the density distribution along rectus femoris cross sectional areas. Each cross section provides a mean HU value. The measured rectus femoris lengths are between 400 and $500 \mathrm{~mm}$ depending from patient anatomy (starting from the pelvis attachment and ending at patellar tendons) the slice increment is $0.625 \mathrm{~mm}$ therefore the number of mean values is between 640 and 800 .

Figure 1 shows the computation results on a healthy subject. It can be noticed how the mean values on the cross sectional areas are rather uniform and displayed in the interval [50-70] HU. Density values are higher at the muscle extremities where the muscle attaches to the tendons.

\section{Modeling muscle fiber direction}

The organization of muscle fiber and tendon in a muscle-tendon architecture is directly connected to the muscle's ability to generate force and movement. Muscle force is in fact proportional to physiologic cross-sectional area (PCSA) while the muscle velocity is proportional to muscle fiber length [18].

A simplified, two dimensional representation of muscle architecture which is commonly used in models of the musculoskeletal system is displayed in Figure 2A (Top figure). The model represents the tendon length, the muscle-fiber length, and the pennation angle. This model assumes that all fiber's have the same length, that all fiber's shorten by the same amount, and that the aponeurosis has the same properties and behavior as the external tendon.

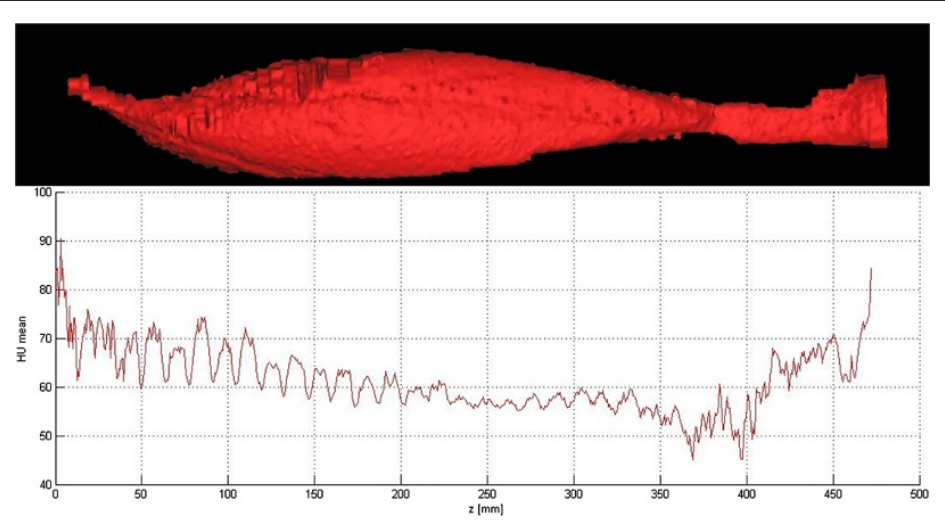

Figure 1 Density distribution along the cross sections in healthy subject (Control). 


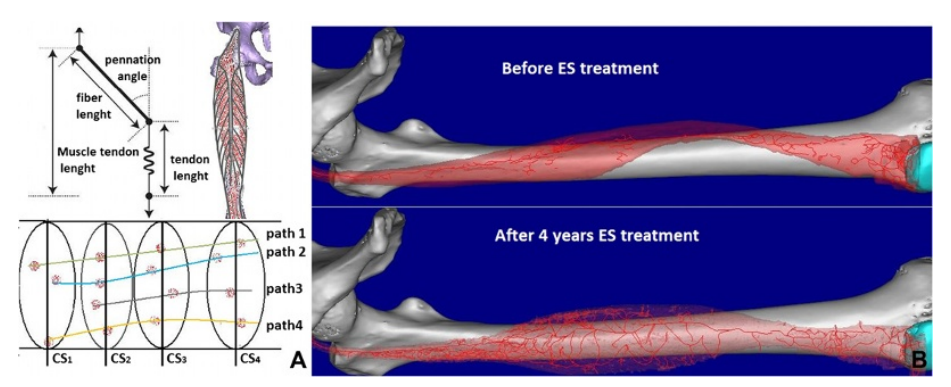

Figure 2 2-Dimensional representation of muscle architecture; the model represents the tendon length, the muscle-fiber length, and the pennation angle (A top-left). Bipennate architecture in Rectus Femoris (A top-right). Schematic representation of density tracker: the points cloud on the cross sections (CS) represents the areas of high density crossed by the paths (A bottom). Central line distribution within RF for patient 2: before ES treatment (B top), after 4 years ES treatment (B bottom).

In quadriceps muscles the architecture is rather complex; the fiber's of the rectus femoris muscle follow three-dimensional trajectories and there are two long aponeuroses distalposterior and proximal-anterior aponeuroses within the muscle (Figure 2A top).

In denervated muscle the fiber direction is not known and a model not yet been developed. It will be reasonable to assume that the restoration process which restores part of the force capability will influence the fiber direction as well.

The muscle fiber are bundled together and surrounded with connective tissue, other tissue such as fat are also present within the muscle but in very small amount. Most of the tissues within a muscle are displayed with values of HU between 30 and 90 . In denervated muscle the degeneration process literally transform the muscle physiology; the muscle is not longer spontaneously contracting and the tissue composition and architecture is modified completely. In terms of HU distribution we have both areas with low and high HU values.

The computational idea is to track the high dense areas on the muscle cross sections in order to create a path along the muscle which passes through the denser muscle regions. We expect different regions on each cross section having this characteristic therefore many paths will be created as seen in Figure 2A (Bottom figure).

The high dense areas on RF are defined experimentally by setting a new threshold to 60$90 \mathrm{HU}$. The paths between such dense areas of the muscle are computed using central line. The central line is defined here as the interpolation of central points through the cross sectional area of a 3D object. The central points on each cross section are unique or multiple depending from the number of edges and from the $\mathrm{HU}$ value. Figure $2 \mathrm{~B}$ shows the central line distribution within a denervated RF at beginning and after 4 years of ES treatment.

\section{Results}

Muscle restoration due to electrical stimulation can be seen quantitatively using the muscle tissue analysis. Muscle undergoing ES treatment are obviously benefiting in terms of mean density, mean densities increase during treatment as seen in Table 2.

Figure 3 shows muscle tissue changes after 4 years of electrical stimulation treatment. Due to stimulation treatment the fraction of normal muscle fibres increases from $45 \%$ 
Table 2 Mean HU density for muscle, in different pathological conditions

\begin{tabular}{lllllll}
\hline Tissue mean Density: HU values & Control & $\begin{array}{l}\text { 1Y } \\
\text { LMN, Pt.1 }\end{array}$ & $\begin{array}{l}\text { 7Y } \\
\text { LMN, Pt. 1 }\end{array}$ & $\begin{array}{l}\mathbf{4} \text { Y } \\
\text { LMN, Pt2 }\end{array}$ & $\begin{array}{l}\mathbf{4} \text { Y LMN +2 } \\
\text { Y ES, Pt.2 }\end{array}$ & $\begin{array}{l}\mathbf{4} \text { Y LMN + } \\
\text { Y ES, Pt.2 }\end{array}$ \\
\hline Muscle: rectus femoris & 54.5 & 45.4 & 32.1 & 35.6 & 42.3 & 46.3 \\
\hline
\end{tabular}

Control (healthy subject) mean density.

Patient 1 mean density: 1 year and after $\mathrm{SCl}$ and 7 years after $\mathrm{SCl}$, the patient was compliant only for the first 1,5 years.

Patient 2 mean density: 4 years after $\mathrm{SCl}, 6$ years after $\mathrm{SCl}$ but with 2 years $\mathrm{ES}$ and 8 years after $\mathrm{SCl}$ with 4 years $\mathrm{ES}$.

to $60 \%$ of the whole volume while connective tissue and fat reduce to $30 \%$ and $50 \%$ respectively.

Figure 4 shows the results from cross sectional density analysis from two RISE patients:

Patient 1 started the ES treatment 1 year after the paralysis. He was compliant to the stimulation protocol for approximately 1.5 years and successively interrupting the treatment. Patient 2 started the ES treatment 4 years after the paralysis. He was compliant over all the monitored period.

- Muscle density increase: Patient 1 and 2 are stimulated in the same way but the stimulation is more effective on patient 1 because muscles at beginning are in better conditions and adipose tissue is thinner as shown in Figure 5 A-B. The area indicated with $a$ in Figure 4 (Patient 1 ) displays a localized increase of density - from 45 to 50 $\mathrm{HU}$ - starting at $120 \mathrm{~mm}$ and ending approximately at $400 \mathrm{~mm}$ from the pelvis attachment. The areas indicated with $d$ and $f$ (Patient 2) displays a localized increase of

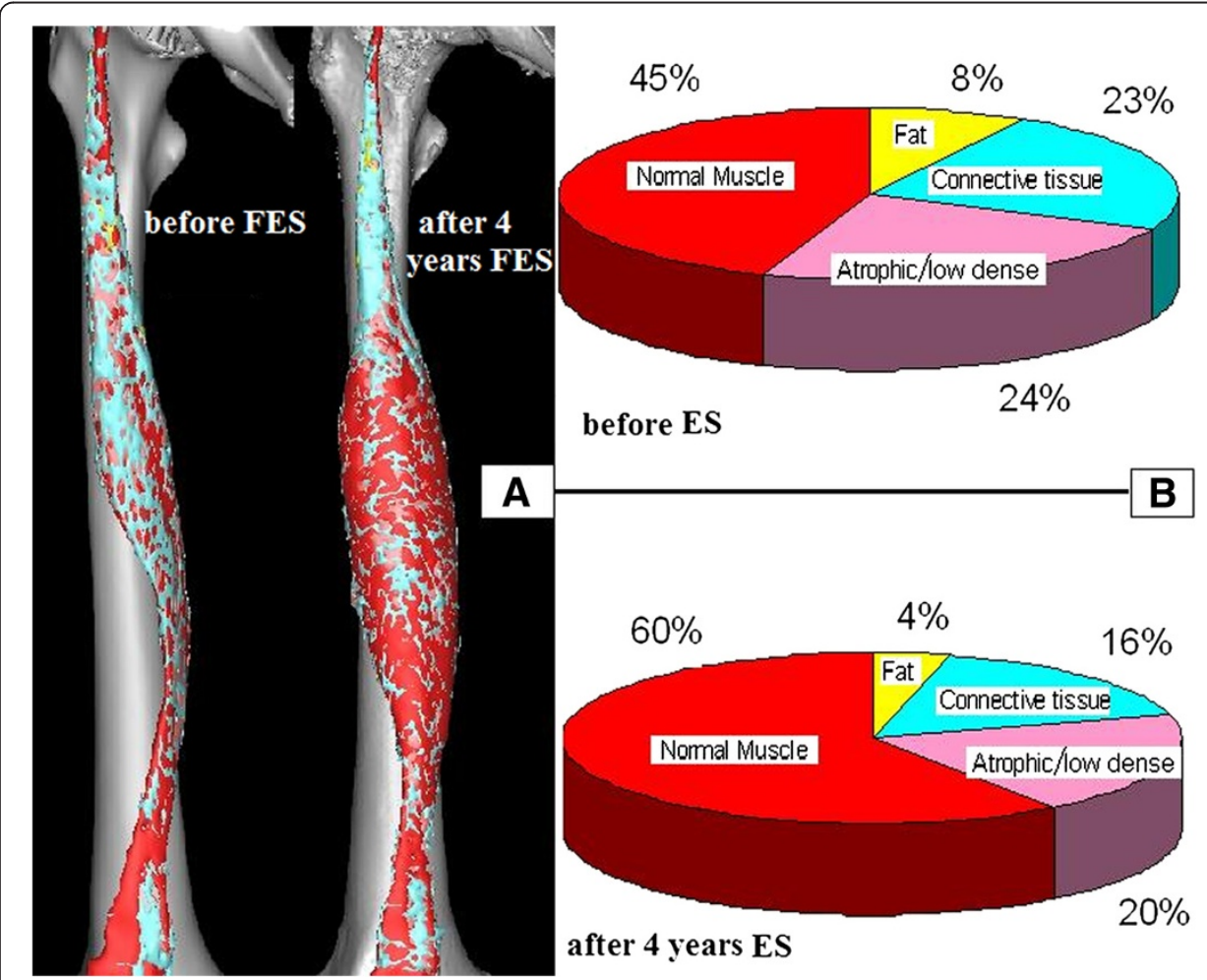

Figure 3 3D models of RF, patient 2 before and after 4 years of ES treatment (A). Chart pies which show the muscle composition at beginning and after 4 years ES according to the Hounsfield intervals established in Table 1 (B). 

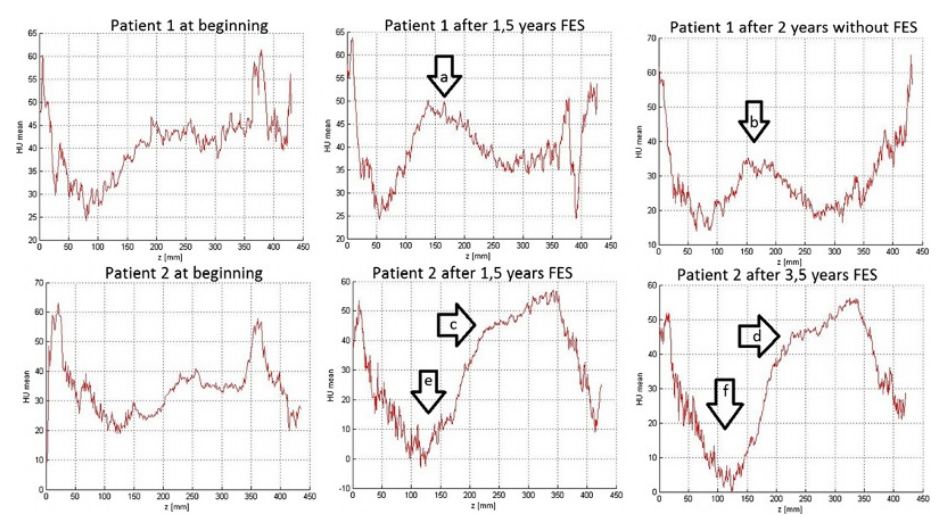

Figure 4 Cross sectional density analysis performed on patient 1 patient 2 before ES treatment, after 1,5 years and after 3,5 years. Patient 1 interrupt the ES treatment after 1,5 years. Patient 2 was compliant during the all period.

density - from 40 to $55 \mathrm{HU}$ - starting at $170 \mathrm{~mm}$ and ending approximately at $350 \mathrm{~mm}$ from the pelvis attachment.

- Muscle density decline in non-compliant patient: comparing the areas indicated with $a$ and $b$ (Patient 1) can be seen a general muscle density decrease of $10 \mathrm{HU}$ over the all length after 2 years of no stimulation.

- Muscle density decline in compliant patient: the areas $c$, e, and $g$ (Patient 2) display zones on rectus femoris between $50 \mathrm{~mm}$ and $150 \mathrm{~mm}$ from the pelvis attachment where muscle density continues to decrease - from 30 down to $5 \mathrm{HU}$ - during the ES treatment. Here the electrical stimulus does not reach efficiently the muscle fibers because the adipose tissue between skin and muscle is too thick (Figure 5B).

The central line algorithm (Computational tool available in MIMICS software [13]) is applied on RF from a compliant patient before and after 4 years ES. The muscle before ES stimulation displays a main central path and many small branches having random
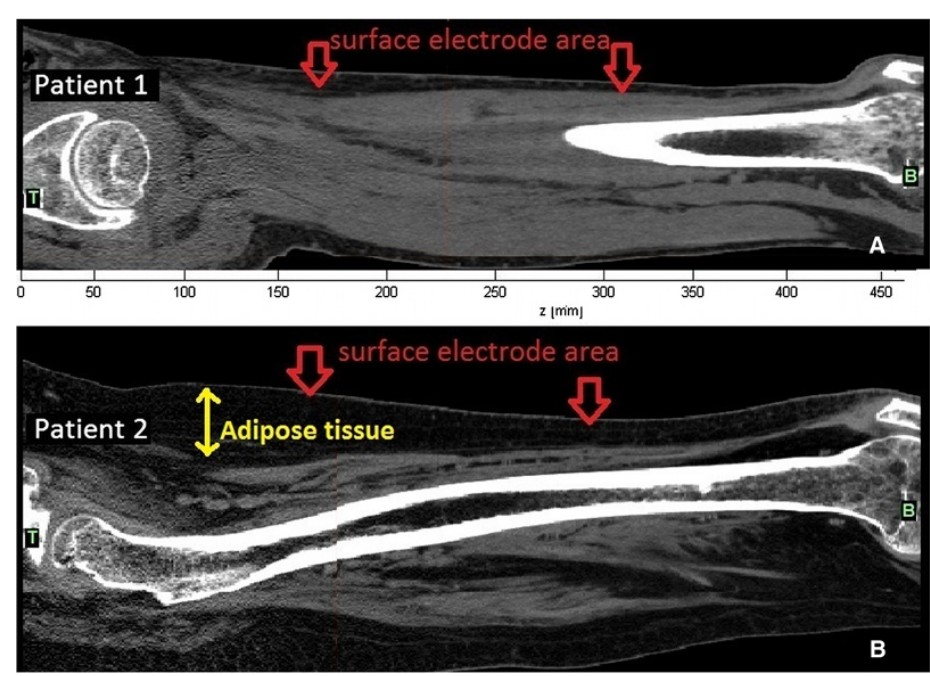

Figure $\mathbf{5}$ Spiral CT pictures (sagittal view) before ES treatment. Patient 1: 1 year after $\mathrm{SCl}$ (A). Patient 2:4 years after $\mathrm{SCl}(\mathbf{B})$. 
directions (Figure 2B top). The central line distribution after 4 years ES has a main central path too, but the numerous branches aren't randomly distributed like in the previous case. Indeed these branches are longer and converge to the centre recalling the bipennate architecture characteristic of rectus femoris (Figure 2B bottom).

\section{Discussion}

The 3D approach combined with muscle tissue analysis provides information on the whole muscle and on its structural changes during ES treatment otherwise not accessible with other monitoring techniques. Muscle growth and tissue restoration can be efficiently monitored. The density analysis along the segmented muscle will also be useful to improve the surface electrode design and its positioning for stimulation. Modeling the muscle fiber direction using central line is possible, further validation is needed but the preliminary results showed here indicate a restoration of bipennate architecture in RF undergoing electrical stimulation treatment. This result confirms the ability of such muscles to recover their functionality and ability to generate force even after long term denervation.

The computational method developed in this work is associated to thresholding criteria and $\mathrm{HU}$ values which are used to define the different tissues within the muscle. Various physical factors can influence the CT number representation during a scan session. The parameter that mostly affects the accuracy and the spatial distribution of HU values is the applied voltage across an X-ray tube; this amplitude is measured in kilo volt $(\mathrm{kV})$ and determines the highest X-ray quantum energy and therefore the attenuation coefficient. CT number distribution is also influenced by phantom (or patient) orientation and position in scan aperture. Therefore it is necessary to know and account for these variabilities when CT numbers are used for tissue characterization and comparison.

\section{Abbreviations}

3D: Three dimensional; SCT: Spiral Computer Tomography; ES: Electrical Stimulation; HU: Hounsfield units; SCI: Spinal Cord Injury; LMN: Lower motor neuron.

\section{Funding sources}

The RISE project was funded by the EU grant (ESB QLG5-CT-2001-02191). This work has been supported by the University Hospital Landspitali research fund

Paolo Gargiulo is assistant professor at Reykjavik University and works as biomedical engineer and researcher at the University Hospital of Iceland. He studied electrical engineering at University Federico II in Naples, and obtained a PhD at Technical University of Vienna, Austria. His research interests are in the field of electrical stimulation medical modeling and rapid prototyping for clinical applications.

Thordur Helgason is associate professor at Reykjavik University and works as biomedical engineer and researcher at the University Hospital of Iceland. He studied electrical engineering at University of Iceland, and obtained a PhD at Technical University of Karlsruhe, Germany. His research interests are in the field of electrical stimulation biomedical technologies.

Páll Ingvarsson is neurologist specialised at Goteborg University, Sweden. He works at Department of Rehabilitation Medicine, Landspitali-University Hospital, Reykjavik, Iceland.

Winfried Mayr is Associate Professor of Biomedical Engineering and Rehabilitation Technology. He works at Medical University of Vienna Center for Medical Physics and Biomedical Engineering. His research interests are in the field of Functional electrical stimulation (mobilization of paraplegics, phrenic pacing, EMG-controlled stimulation, pelvic floor applications, denervated muscles, application of FES in microgravity and bed-rest) Implant technology (Microelectronical and electromechanical implants).

Ugo Carraro is Associate Professor of General Pathology at the Faculty of Medicine of the University of Padova, since 1983. Editor-in-Chief of Basic and Applied Myology/The European Journal of Translational Myology since 1991, he founded and chair since 2005 the University of Padova Interdepartmental Research Center of Myology. Helmut Kern is head of the "Department of Physical Medicine and Rehabilitation of the Wilhelminenspital" (Vienna, Austria) since 1984 and director of the research institute "Ludwig Boltzmann Institute of Electrical Stimulation and Rehabilitation" (Vienna, Austria) since 1988.
} 


\section{Authors' Contributions}

PG and TH developed the monitoring techniques described in this paper. PI supervised the clinical trial in Iceland and participates in designing the monitoring strategies. WM is the project leader and main developer for the electrical stimulators employed in the project. HK participated in the design of the study and the patient's recruitment. UC carried out the histological studies on the muscles and helped to interpret the results. All authors read and approved the final manuscript.

\section{Acknowledgments}

This work has been supported by the University Hospital Landspitali research fund

\section{Author details}

'Department of Development and Consultancy HUT, University Hospital Landspitali, Armuli 1A, Reykjavik 108, Iceland. ${ }^{2}$ Department of Biomedical Engineering, University of Reykjavik, Armuli 1A, Reykjavik 108, Iceland. ${ }^{3}$ Department of Rehabilitation Medicine, Landspitali-University Hospital, Reykjavik, Iceland. ${ }^{4}$ Medical University of Vienna, Center of Biomedical Engineering and Physics, Vienna, Austria. ${ }^{5}$ Ludwig Boltzmann Institute of Electrostimulation and Physical Rehabilitation, Department of Physical Medicine, Wilhelminenspital, Vienna, Austria. ${ }^{6}$ Department of Biomedical Sciences, Laboratory of Translational Myology of the University of Padova, Padova, Italy.

Received: 17 January 2012 Accepted: 12 April 2012

Published: 24 April 2012

\section{References}

1. Fisher AL (2004) Of worms and women: sarcopenia and its role in disability and mortality. J Am Geriatr Soc 52:1185-1190

2. Janssen I, Heymsfield SB, Ross R (2002) Low relative skeletal muscle mass (sarcopenia) in older persons is associated with functional impairment and physical disability. J Am Geriatr Soc 50:889-896

3. Kern H, Hofer C, Strohhofer M, Mayr W, Richter W, Stöhr H (1999) Standing up with denervated muscles in humans using functional electrical stimulation. Artif Organs 23(5):447-452

4. Kern H, Hofer C, Mödlin M, Mayr W, Vindigni V, Zampieri S, Boncompagni S, Protasi F, Carraro U (2007) Stable muscle atrophy in long-term paraplegics with complete upper motor neuron lesion from 3- to 20-year SCI. Spinal Cord 46:293-304

5. Mayr W, Bijak M, Rafolt D, Sauermann S, Unger E, Lanmuller H (2001) Basic design and construction of the Vienna ES implants: existing solutions and prospects for new generations of implants. Med Eng Phys 23:53-60

6. Kern H, Hofer C, Mayr W, Carraro U (2009) European Project RISE: Partners, protocols, demography. Basic Appl Myol 19:211-216

7. Mödlin M, Forstner C, Hofer C, Mayr W, Richter W, Carraro U, Protasi F, Kern H (2005) Electrical stimulation of denervated muscles: first results of a clinical study. Artif Organs 29:203-206

8. Kern H, Carraro U, Adami N et al (2010) One year of home-based Functional Electrical Stimulation (ES) in complete lower motor neuron paraplegia: recovery of tetanic contractility drives the structural improvements of denervated muscle. Neurol Res 32:26-32

9. Bizzarini E, Zampa A, Malisan C, Pinzini C, Di Benedetto P, Kern H, Adami N, Carraro U (2009) Epidemiology and clinical management of Conus-Cauda Syndrome and flaccid paraplegia in Friuli Venezia Giulia: Data of the Spinal Unit of Udine. Basic Appl Myol 19:163-167

10. Boncompagni S, Kern H, Hofer C, Mayr W, Carraro U, Protasi F (2007) Structural differentiation of skeletal muscle fibers in the absence of innervation in humans. Proc Natl Acad Sci Unit States Am 104:19339-19344

11. Lotta S, Scelsi R, Alfonsi E, Saitta A, Nicolotti D, Epifani P, Carraro U (1991) Morphometric and neurophysiological analysis of skeletal muscle in paraplegic patients with traumatic cord lesion. Paraplegia 29:247-252

12. Hofer C, Forstner C, Mödlin M, Jäger H, Mayr W, Kern H (2005) In vivo assessment of conduction velocity and refractory period of denervated muscle fibers. Artif Organs 29(6):436-439

13. Gargiulo P, Helgason T, Reynisson PJ, Helgason B, Kern H, Mayr W, Ingvarsson P, Carraro U (2011) Monitoring of Muscle and Bone Recovery in Spinal Cord Injury Patients Treated With ES Using Three-Dimensional Imaging and Segmentation Techniques: Methodological Assessment. Artificial Organs 35(3):275-281

14. Mandl T, Meyerspeer M, Reichel M, Helmut K, Hofer C, Mayr W (2008) Functional electrical stimulation of longterm denervated, degenerated human skeletal muscle: estimating activation using T2-parameter magnetic resonance imaging methods. Artif Organs 32(8):604-608

15. Gargiulo P, Vatnsdal B, Ingvarsson P, Knútsdóttir S, Gudmundsdóttir V, Yngvason S, Helgason T (2008) Restoration of Muscle Volume and Shape Induced by Electrical Stimulation of Denervated Degenerated Muscles: Qualitative and Quantitative Measurement of Changes in Rectus Femoris Using Computer Tomography and Image Segmentation. Artif Organs 32:609-613

16. Gargiulo P, Kern H, Carraro B, Vatnsdal U, Ingvarsson P, Knútsdóttir S, Gudmundsdóttir V, Yngvason S, Helgason T (2010) Quantitative color 3-dimensional computer tomography imaging of human long-term denervated Muscle. Neurological Research 32:13-20

17. Materialise. Product: MIMICS. Available at: http://www. materialise.com. Accessed April 3, 2012.

18. Blemker SS, Delp SL (2006) Rectus femoris and vastus intermedius fiber excursions predicted by three-dimensional muscle models. J Biomech 39(8):1383-1391

doi:10.1186/2192-1962-2-10

Cite this article as: Gargiulo et al: Medical image analysis and 3-d modeling to quantify changes and functional restoration in denervated muscle undergoing electrical stimulation treatment. Human-centric Computing and Information Sciences 2012 2:10. 